\title{
UNE NOUVELLE APPROCHE DE L'ESPACE PUBLIC
}

\author{
Eric Dacheux ${ }^{1}$
}

Qu'est-ce que la démocratie ? Un idéal, nous rappelle Jacques Rancière, celui de l'égalité et de l'autonomie, chacun en démocratie peut exercer le pouvoir sans condition de classe, de race, de religion ou de savoir, comme le signifie la pratique antique du choix du gouvernement par tirage au sort (Rancière, 2005). Mais cet idéal, que la démocratie poursuit sans jamais l'atteindre, se double d'institutions et de règles concrètes : élection de représentants, séparation des pouvoirs, vote à la majorité, État de droit, etc. : «La démocratie est la procédure, dans sa version représentative, par laquelle les gouvernés gouvernent, désignent et sanctionnent les gouvernants »(Leca, 2004). Cependant, la démocratie, c'est aussi un régime politique particulier marqué par l'instauration d'un espace de médiation entre la société civile et l'État qui favorise, par le débat contradictoire, l'émergence d'une opinion publique. Cet espace - qui n'existe pas dans les régimes totalitaires - c'est l'espace public. Cette notion reste marquée par celui qui l'a popularisée : Jurgen Habermas(1978). Pourtant, il ne s'agit pas, ici, de montrer l'évolution de la pensée de cet auteur, de confronter cette pensée à d'autres grands penseurs de cette notion (par exemple Arendt ou Senett), de synthétiser les différentes critiques adressées à cet

1 Professeur des universités en sciences de l'information et de la communication, Université Blaise Pascal, responsable du groupe de recherche «communication et solidarité », LRL, MSH Clermont Fd.

Recherches en communication, $\mathrm{n}^{\circ} 28$ (2007). 
auteur, ni même de chercher à valider empiriquement tel ou tel aspect de la théorie habermassienne. Non, même si toutes ces voies sont pertinentes, elles sont également très souvent empruntées, c'est pourquoi nous voudrions, ici, proposer une autre approche épistémologique : comprendre la nature de la société démocratique (première partie) pour cerner les trois dimensions de l'espace public (seconde partie)

\section{La démocratie un compromis historique}

Pour certains, société démocratique et société capitaliste ne font qu'un. C'est le cas de Gary Stanley Becker. En effet, pour ce prix Nobel d'économie, le social tout entier peut s'expliquer par le calcul rationnel de l'individu'. Tout choix, même celui qui est apparemment le plus irrationnel est, en réalité, le fruit d'un coût objectif qu'il suffit de déterminer (le criminel va, par exemple mesurer le gain du crime au coût de la peine prévue pour ce crime). En effet, la vie humaine, explique Becker, c'est du temps. Or, le temps est une ressource rare qu'il convient donc de maximiser. Chaque action humaine a donc un coût (le temps passé à cette action) et par conséquent chaque activité humaine est décidée d'après un calcul d'opportunité visant à définir si l'action doit être ou non entreprise. L'individu est donc une entreprise qui maximise son capital (humain) sous contrainte de temps. Dans ce cadre d'analyse, la dimension économique, la dimension politique et la dimension symbolique sont confondues. La société démocratique n'est qu'un vaste marché constitué par l'ensemble des interactions utilitaristes. Bien entendu, de nombreux chercheurs et de nombreux acteurs combattent une conception si réductrice de la démocratie. Certains se réclament du libéralisme politique et mettent alors en avant une conception minimaliste de l'espace public, d'autres rappelant la complexité du social, soulignent, au contraire, la richesse de l'espace public...

\section{Critique de la vision libérale de l'espace public}

L'équation démocratie = libéralisme politique que l'on retrouve dans la bouche de nombreux acteurs politiques ne va pas de soit. En effet, les grands noms qui se rattachent au libéralisme politique (Kant,

1 Voir, par exemple, G.S. Becker, The Economic Approach to Human Behavior, Chigaco, The university of Chicago Press. 
Tocqueville, Constant, Mills, etc.) ne doivent pas faire oublier que les libéraux développent une conception de la démocratie qui peut être critiquée par les théoriciens de la démocratie. Elle est ainsi, dans la lignée de Platon et de Montesquieu, remise en cause par ceux qui opposent la sagesse du savant à l'ignorance du peuple. Par exemple, pour Schumpeter (1969), la complexité des problèmes contemporains exclut le développement d'une démocratie participative qui serait exercée par des citoyens qui n'ont pas les compétences nécessaires pour fonder une opinion rationnelle et qui, par conséquent, sont facilement manipulés par des leaders plus ou moins démagogiques. Cette vision élitiste aboutit à une conception technocratique de la vie démocratique qui doit être gérée, loin des passions partisanes, par des professionnels compétents choisis par les électeurs.

Face à cette vision élitiste, il est bien évident que l'héritage des Lumières conjuguant autonomie du sujet et libre exercice de la raison par tous les citoyens qu'incarne le libéralisme politique doit être assumé. Mais cet héritage qui se traduit, dans les faits, par une démocratie représentative où des citoyens élisent périodiquement les élites qui les gouvernent, peut être dépassé. En effet, dans l'idéal libéral tel qu'exprimé par Isaiah Berlin (1969), toutes les idées sont équivalentes et doivent être respectées. Dans ces conditions, la tolérance consiste à ne pas défendre publiquement ses idées comme étant supérieures aux autres. Dans une telle vision, l'Etat doit être neutre, il ne doit pas promouvoir de valeurs particulières, mais, par sa neutralité même, garantir un consensus minimal favorisant la gestion et la cohabitation de conceptions différentes. Les débats deviennent alors essentiellement une affaire privée et la volonté populaire n'est que l'agrégation des préférences individuelles qui se sont formées au sein de la sphère privée. L'espace public n'est donc pas le lieu où se développe un débat public permettant la formation d'une opinion publique, mais le lieu de la publicisation du résultat de l'agrégation des opinions individuelles. Cette formation du « consensus par recoupement » cher au premier Rawls exclut donc le débat public, facteur de conflit entre conceptions différentes et légitimes de l'intérêt général, au nom d'une neutralité seule à même de garantir le maintien de la pluralité des points de vue.

Evidemment, cette conception minimaliste de l'espace public est critiquée. Pour des raisons empiriques évidentes (la controverse est présente dans l'espace public) comme pour des raisons théoriques majeures (la démocratie n'est pas seulement un droit c'est aussi un devoir de participation). Il faut donc développer une conception plus 
riche, multidimensionnelle, de l'espace public. Comment ? Jürgen Habermas propose une voie : la synthèse théorique. En effet, comme il l'explique parfaitement lui-même (chapitre 7 de «Droit et Démocratie»), sa conception de la démocratie délibérative (et donc sa vision de l'espace public politique) représente une synthèse entre trois visions de la démocratie. La première, libérale, est axée sur les droits individuels, tandis que la seconde, républicaine, est basée sur le devoir de participation politique. La troisième est systémique et met l'accent sur la division inexorable de nos sociétés complexes en sous-systèmes autopoiétiques se régulant indépendamment des citoyens. Ces trois théories ne sont pas forcément complémentaires, pourtant Habermas emprunte à chacune d'elle. Selon lui, l'espace public « ne peut pas se concevoir comme une institution, ni, assurément, comme une organisation [...]. Il ne constitue pas non plus un système; il admet certaines frontières intérieures, mais, vis-à-vis de l'extérieur, se caractérise par des horizons ouverts, poreux et mobiles » (Habermas, 1997, p.387). Au sein de ces frontières poreuses, « la procédure démocratique de législation est conçue de telle sorte que les citoyens fassent de leurs droits de communication et de participation un usage qui soit, entre autres, orienté vers le bien public, usage que l'on peut demander, mais qui ne peut être obtenu de force par le droit » (Habermas, 1997, p. 492). Loin de cette élégante synthèse habermassienne, nous proposons une autre approche de la démocratie resituant, elle aussi, toute la complexité théorique et empirique de l'espace public.

\section{La démocratie, un compromis historique entre trois ordres infrastructurels}

Dans un cadre d'analyse qui doit beaucoup à Lefort (1986) et Castoriadis (1975), nous pensons que la démocratie est un dessein qui se fonde sur lui-même. Pour se transformer et perdurer, ce dessein doit identifier les constituants qui le fondent. Les constituants et non pas «le » constituant, le principe essentiel et unique d'où jaillirait la vie sociale. En effet, contrairement à de nombreux penseurs ayant cherché à identifier le fondement ultime du social (le politique pour Aristote et Hobbes, l'économique pour Marx ou Hayek, le symbolique pour Mauss ou Lévi-Strauss) nous pensons, à la suite de Fernand Braudel (1979), que la société est «l'ensemble des ensembles », un jeu d'alliances et 
d'oppositions entre des systèmes ayant leur logique propre ${ }^{1}$. Dans cette perspective théorique, nous rejoignons Eric Forgues (2000) pour qui toute société humaine résulte de l'interaction conflictuelle entre trois axes infrastructurels, trois ordres fondamentaux : l'économique, le politique, le symbolique ${ }^{2}$. Suivons le raisonnement de cet auteur. Dans un premier temps, Forgues rappelle l'apport de Marx : "Chez Marx, la formation sociale et celle de la conscience renvoient en dernière analyse au développement des forces productives dont la motivation anthropologique fondamentale tire son origine de la nécessité de reproduire les conditions matérielles de l'existence » (p. 190). Dans un second temps, le chercheur québécois met en lumière la filiation marxiste d'Habermas que les tenants de la démocratie délibérative semblent trop souvent occulter. "Si Habermas reconnaît cette exigence [la reproduction des conditions matérielles de l'existence] et le rôle qu'elle joue dans le développement de la rationalité technique, il critique cette idée que seul le développement des forces productives détermine et explique les transformations sociales (1973, 1978, 1985). [...]. Habermas (1987b) met plutôt en évidence la spécificité et l'irréductibilité des exigences normatives, déterminées au sein de l'espace public et des activités communicationnelles, dans la transformation des rapports sociaux » (p. 191). Face à une rationalité instrumentale qui cherche à coordonner les actions en vue de maîtriser la nature dans la sphère économique et à légitimer le pouvoir en place dans la sphère politique, se déploie une rationalité communicationnelle qui vise à l'intercompréhension dans le monde vécu. Il y a donc deux rationalités, deux axes infrastructurels déterminant la formation d'une société : le développement de la technique (rationalité instrumentale) et celui des normes (raison communicationnelle). Dans un troisième temps, Forgues revient sur le tournant linguistique (linguistic turn) de la philosophie en générale et celle de Jürgen Habermas en particulier. Contre la philosophie Kantienne

1 L'historien distingue quatre ensembles composant la société : la hiérarchie sociale, la civilisation (ou culture, pour lui ces deux termes sont équivalents), l'Etat, l'économie (Braudel, 1979, T2, chp.5).

2 On peut également interpréter la réflexion de Braudel dans ce sens, puisque, dans la conclusion du troisième et dernier tome de «Civilisation matérielle et capitalisme », il définit trois types de hiérarchie sociale ( « [...]celles de l'argent, celles de l'Etat, celles de la culture [...] ») qui s'affrontent (Braudel, 1979, T3, p. 540). Cette remarque peut laisser entendre que l'ordre social (la société), provient de l'interaction conflictuelle entre l'économique (l'argent), le politique (l'Etat) et le symbolique (la culture). 
et contre la sociologie marxiste, Habermas va inscrire la rationalité non plus dans le sujet ou dans la technique, mais dans la communication intersubjective ; communication cependant réduite au langage, à une structure sociale déjà présente qui s'impose aux individus. Cette réduction de la communication au langage réduit le symbolique. Or, d'une part, les travaux en sciences de la communication (que ne cite pas l'auteur), soulignent l'importance du non verbal et du para verbal dans les communications humaines et montrent ainsi que l'échange rationnel ne peut jamais complètement échapper ni à l'émotion esthétique ni à l'influence rhétorique, tandis que l'anthropologie de l'imaginaire (que l'auteur convoque) met en lumière l'importance empirique des images dans notre rapport au monde et remet en cause, d'un point de vue théorique, la réduction du symbole au signe. Au terme de sa démonstration, Eric Forgues peut donc conclure : «Si Marx a permis de comprendre la société à travers le développement de l'infrastructure technique, Habermas complète cette compréhension en montrant que le développement de la technique ne détermine pas seul les transformations sociales, puisque le développement des structures normatives sous-tendant l'interaction les détermine également. Parallèlement à ces deux axes infrastructurels, nous voulons intégrer l'axe symbolique dont le développement détermine également les transformations sociales à travers les possibilités "individuantes » qu'offrent les ressources symboliques et la structure de conscience » (p. 211).

Bien sûr, notre propos n'est pas le même que celui de Forgues. Il ne s'agit pas d'identifier trois types de rationalité afin de justifier la permanence du religieux dans nos sociétés. Notre volonté est plutôt de souligner combien il est nécessaire, pour penser les évolutions des sociétés démocratiques, de prendre en compte l'intrication complémentaire et conflictuelle de trois ordres qui se déterminent réciproquement : l'économique (la mise en valeur des ressources), le politique (l'élaboration des normes) et le symbolique (la construction du croire). Dans cette construction théorique novatrice, la démocratie est, simultanément, un régime politique particulier (marqué par l'instauration d'un espace public) et une société singulière (fondée sur elle-même). Ainsi deux éléments constituent la démocratie moderne. D'une part, une interaction antagoniste particulière entre ces trois ordres qui fait, à un moment 
particulier de l'histoire, surgir un espace public'. D'autre part, une nouvelle régulation de cette interaction antagoniste qui, justement, se joue désormais, en grande partie, dans l'espace public. L'espace public est donc à la fois le fruit d'un compromis particulier, historiquement situé, entre les trois ordres, et l'instance, propre à la démocratie, où se régule, depuis lors, le conflit entre ces trois ordres. L'espace public n'est donc pas une donnée a-historique, mais un espace évolutif, propre à la modernité, traversé de tensions contradictoires, où se fonde et se refonde la démocratie au risque, à chaque fois, de se dissoudre.

\section{Les trois dimensions de l'espace public}

Dans la Haine de la démocratie, Jacques Rancière (2005) nous met en garde contre deux dangers : celui de réduire la démocratie à un mode de gouvernement particulier (la domination d'une majorité - la plus forte minorité dit Rancière) et celui d'étendre la démocratie à l'ensemble des relations socio-économiques régissant la société (la société capitaliste). Si effectivement nous pensons, avec Rancière, que la démocratie ne doit être confondue ni avec un mécanisme de reproduction du pouvoir au main de la bourgeoisie ni avec un système capitaliste se développant d'ailleurs fort bien en régime autoritaire (cf. la Chine), nous pensons, sans lui, que la démocratie est un compromis social historique ayant engendré un régime politique particulier marqué par l'instauration d'un espace public. Elle est donc à la fois une modalité « d'être ensemble » spécifique (elle est fondée sur elle-même et vise l'égalité entre altérité radicale) et une forme particulière d'exercice du pouvoir basé sur la nécessité de légitimer la norme par le débat public. Telle est, selon nous, la double nature de la démocratie. Cette double nature permet de mieux cerner les trois dimensions de l'espace public : politique, symbolique et économique

1 Pour simplifier et à titre d'exemple, l'espace public surgit en France, au XVIIi ìme siècle, à une époque marquée, entre autres, par la révolution (ordre politique), les Lumières (ordre symbolique) et la naissance du capitalisme industriel (ordre économique). 


\section{La dimension politique de l'espace public}

Pour Claude Lefort, la politique, c'est-à-dire la mise en œuvre du pouvoir, objet disciplinaire construit par la science politique et la sociologie politique, n'est qu'une faible partie du politique défini comme la " mise en forme », c'est-à-dire précise Lefort, «la mise en scène et la mise en sens », qui institue le social (Lefort, 1986, p. 256-258). Pour notre part, l'ordre politique est celui de la construction de la norme. Cette construction, comme le souligne Habermas lui-même, ne s'effectue pas uniquement dans l'espace public. Tout d'abord, la norme s'élabore loin des regards publics, c'est-à-dire dans l'espace calme et discret des couloirs des assemblées, dans le secret des conseils ministériels, dans ces lieux réservés où, loin des débats publics, on prend seuls la décision définitive. Cet espace, c'est l'espace politique (Wolton, 1991) qui constitue la limite haute à l'espace public, Ensuite, comme l'indique nettement Lefort et comme le précisent aussi des chercheurs en communication (Dalgreen, 2000 ; Garnham 1992) et comme d'ailleurs l'indiquait Habermas dans «L"espace public », l'opinion que chacun peut se forger des problèmes de la société, se forge aussi, dans l'espace domestique via, aujourd'hui, les mass media (Habermas, 1978). Il existe donc un soubassement, une limite basse à l'espace public même si cette limite, comme l'indique Bernard Floris (2003), est difficile à percevoir car fluctuante. Ces trois espaces (politique, public et domestique) sont connectés grâce à l'espace médiatique qui les relie tous les trois, mais aussi par des espaces de médiation spécifiques qui les rassemblent deux par deux. D'une part, l'espace de institutionnel de médiation qui relie, l'espace politique à l'espace public et, d'autre part, l'espace civil (Floris 2003) qui relie l'espace politique à l'espace domestique (cf. tableau

$\mathrm{N}^{\circ} 1$ ). Chacun de ces espaces prend des formes empiriques distinctes :

- Les dispositifs participatifs. Ce sont des espaces intermédiaires entre l'espace politique et l'espace public. Ils permettant à une institution d'entrer en contact directement avec les citoyens ou les représentants de collectifs issus de la société civile. Ces dispositifs répondent, dans des proportions variables, a plusieurs des fonction suivantes : l'information (la publicité au sens kantien du terme), la consultation (interrogation des personnes concernées par un projet de l'institution), la concertation (réflexion conjointe), la suggestion (recueil de propositions de projets ne venant pas de l'institution mais susceptible d'être mise en place par elle), etc. 
- Les arènes. Ce sont des espaces intermédiaires entre l'espace domestique et l'espace public. Ce sont donc des espaces de médiation propre à la société civile. Contrairement aux espaces d'interconnaissance ${ }^{1}$ où le lien social est souvent la condition première de l'échange, ces espaces obéissent à une volonté (souvent celles d'un tiers, des militants associatifs, par exemple) de créer les conditions de l'échange entre des personnes ne se rencontrant que fort peu. Il s'agit tout à la fois de relier, de constituer un collectif, mais également de séparer, de maintenir la distance critique individuelle. Ces arènes combinent, de manière variée, plusieurs logiques : l'expression des personnes marginalisées et/ ou prisonnières de leur sphère domestique, la conciliation entre acteurs, la reconnaissance de populations stigmatisées, la critique de décisions politiques, etc.

\section{Les espaces du politique (Tableau $\mathbf{N}^{\circ} \mathbf{1}$ )}

\begin{tabular}{|l|c|}
\hline \multicolumn{1}{|c|}{ DENOMINATIONS THEORIQUES } & EXEMPLES EMPIRIQUES \\
\hline Espace politique & Conseil des ministres \\
\hline \hline Espace institutionnel de médiation & $\begin{array}{c}\text { Dispositif participatif } \\
\text { Conseil local de développement }\end{array}$ \\
\hline Espace public & Manifestation de rue \\
\hline Espace civil & $\begin{array}{c}\text { Arène } \\
\text { Forum social }\end{array}$ \\
\hline \hline Espace domestique & Foyer \\
\hline \hline
\end{tabular}

1 Dans ces espaces d'interconnaissances, la confrontation d'idées (qui ne sont pas forcément politiques, mais qui peuvent l'être) est privée, mais elle est également publique, puisque elle se déroule dans un espace social non domestique et donc potentiellement accessible à d'autres personnes. En réalité, cette potentialité est limitée parce que, dans ces espaces (bars, marchés, machine à café d'une entreprise, etc.), l'interconnaissance est très souvent l'une des conditions nécessaires de l'échange. 
Cette distinction entre dispositif participatif et arène n'épuise certainement pas la diversité des logiques constitutives des espaces intermédiaires. De plus, elle propose un découpage analytique dichotomique tranché (entre logiques propres à la société civile et logiques institutionnelles), alors que, dans la réalité empirique, les choses sont souvent plus complexes, plus mouvantes. Les logiques de l'arène et du dispositif participatif migrent parfois d'un espace intermédiaire à l'autre, à l'image des budgets participatifs qui sont aujourd'hui adoptés par certaines municipalités. Malgré tout, cette distinction permet, d'une part, de mettre en lumière la pluralité des lieux de débats et des logiques d'acteurs dans l'ordre politique et, d'autre part, de rappeler que la construction de la norme de se réduit pas à un processus langagier entre acteurs rationnels. Du coup, loin d'une vision libérale, ce qui est mis en avant, ce sont les tensions politiques structurantes de l'espace public. Tensions entre fragmentation (des arènes, des dispositifs participatifs, des médias, etc. ${ }^{1}$ ) et homogénéisation (recours à la communication marketing ${ }^{2}$ ), entre renouveau de l'engagement politique (via les associations) et repli sur la sphère domestique, entre ouverture sur l'international et centration sur l'espace local, etc.

\section{La dimension symbolique de l'espace public}

L'ordre symbolique est celui de la circulation du croire. Définition qui s'éloigne de la définition classique. Dans l'acception la plus courante - celle que Lévi-Strauss puis Lacan ont donné à la suite des travaux de Marcel Mauss (Tarot, 2003) - le symbolique, c'est ce qui donne sens à la société. Plus précisément, le symbolique est la société qui se comprend elle-même, qui prend conscience d'elle-même. Le symbolique est la réalité sociale telle que nous la percevons. Mais si le symbolique fonde un ordre propre, c'est que les symboles ne renvoient pas uniquement à un référent, mais s' articulent les uns aux autres dans un réseau complexe de significations. Le symbolique est donc un réseau de significations que l'individu va intérioriser au cours de la socialisation, une structure sociale inconsciente qui va déterminer sa manière de comprendre le monde. Une telle définition a l'avantage de souligner la persistance du symbolique dans nos sociétés. Cependant, comme le relève justement Castoriadis (1975), cette approche classique a tendance à figer le social,

1 Cf. François, Neveu, 1999.

2 Cf. Miège, 1989. 
à soumettre l'individu à des structures symboliques qui lui échappent et le déterminent entièrement. Notre vision du symbolique est plus dynamique. Selon nous, le symbolique est l'articulation de constructions ${ }^{1}$ qui s'engendrent mutuellement, s'opposent, s'entrelacent. Loin d'imposer le primat absolu d'une instance symbolique sur l'autre (la raison sur le sacré, par exemple), nous les pensons en déséquilibre ; loin de les considérer comme étanches les unes autres, nous voulons mettre en lumière leurs influences réciproques. Ni domination du logos ou de l'image, ni primauté du sacré ou du profane ni règne hégémonique de la pulsion ou empire de la raison, mais conflits permanents et emprunts réciproques entre toutes ces constructions symboliques appartenant à deux pôles opposés : le pôle autonome du symbolique constitué de constructions immanentes (science, idéologie, utopie, etc.) et le pôle hétéronome composé de constructions qui font référence à la transcendance (religion, cosmogonie, mythologie, etc.). Il y a, bien sûr, des oppositions fortes entre ces deux pôles (entre science et religion, par exemple), mais aussi une grande porosité (des sciences se constituent autour de l'objet religion, la religion trouve dans la science de quoi mieux se comprendre, voire de quoi se justifier ${ }^{2}$ ).

Contrairement à la dimension politique qui, nous l'avons vu, est marqué par une pluralité de logiques engendrant des tensions dynamiques, la dimension symbolique de l'espace public est caractérisée par une panne : l'absence d'un conflit intégrateur entre idéologie et utopie. Il faut penser l'utopie et l'idéologie non pas isolément, mais comme le démontre Paul Ricoeur, dans leur rapport dialectique (Ricoeur, 1997). Selon lui, l'idéologie et l'utopie sont des structures ternaires qui se répondent et s'opposent. A un premier niveau, qu'il nomme pathologique, l'idéologie est une dissimulation, une distorsion de la réalité, tandis que l'utopie est une fuite hors de la réalité. A un second niveau, l'idéologie a pour fonction de légitimer l'autorité établie, au risque d'une distorsion de la réalité. Risque que combat l'utopie, puisque, dit Ricoeur, elle a pour fonction d'exposer le problème de crédibilité des

1 Nous utilisons le mot « construction » plutôt que les mots « système » ou « forme » généralement usités. D’une part, pour renvoyer à une intentionnalité immanente, même si le sens de ces constructions échappe en partie aux intentions humaines qui ont présidé à leur constitution ; d'autre part, pour inscrire ces « constructions » dans la conscience collective, même si elles s'inscrivent également dans l'inconscient collectif. Ces constructions sont, par définition, collectives, partagées, même si elles sont quelque fois référées à un fondateur unique.

2 Comme le célèbre « Dieu ne joue pas aux dés » d'Einstein. 
systèmes d'autorité. Enfin, à un troisième niveau, l'idéologie est une matrice de l'intégration sociale, alors que l'utopie, en proposant des variations imaginaires sur le pouvoir, le gouvernement, la famille ou la religion est une forme de subversion sociale. On le voit, il s'agit bien de penser une tension, un équilibre instable, à chacun des trois niveaux entre utopie et idéologie. La recherche de l'équilibre entre utopie et idéologie fortifie la démocratie, mais si le déséquilibre est trop fort, la démocratie vacille. C'est alors que surgit la nostalgie. En effet, en l'absence d'une utopie constituée perdure la contestation de l'idéologie. Mais cette dimension critique présente dans l'utopie, n'est plus encadrée par les dimensions oniriques et rationnelles permettant d'imaginer les contours d'un autre monde désirable. Dès lors, la contestation se fait souvent au nom d'un retour chimérique à un monde ancien, un âge d'or, un paradis perdu, un entre soi qu'il faut retrouver. Ce qui se traduit par le développement d'une "identité refuge » (Wolton, 2005) unidimensionnelle (la nationalité par exemple) qui conduit à rejeter l'autre.

\section{La dimension économique de l'espace public}

L'ordre économique est celui de la mise en valeur des ressources. Pour survivre et s'épanouir, les sociétés humaines organisent la production, la circulation et la consommation des ressources naturelles (faune, flore, etc.), humaines et artificielles (objets, savoirs scientifiques, etc.). Dans cette perspective, l'ordre économique ne se réduit pas au marché auto régulateur cher à Hayek. L'ordre économique est régi par une pluralité de principes d'échanges (le marché, mais aussi la redistribution et la réciprocité) et des modes de production (artisanal, mutualiste, capitaliste, etc.) L'ordre économique, si l'on en croit Braudel (1979) et Polanyi (1983), fut longtemps dominé par les deux autres. Cependant, les relations entre les trois ordres ne sont pas stables et, nous dit l'historien, aucun ordre n'a toujours le pas sur les autres. A partir du XI ème siècle, affirme Braudel, la domination de l'ensemble « économie » sur les autres ensembles se fait de plus en plus nette ${ }^{1}$. Elle se renforce au fur et à mesure que l'économie

1 Dans le chapitre V du tome II de «Civilisation matérielle et capitalisme », Braudel définit la société comme l'ensemble des ensembles : l'économique, le social hiérarchique, le politique, le culturel. Pour lui, il convient de garder cette pluralité à l'esprit mais «plus encore, il importe de ne pas imaginer a priori que tel ou tel secteur puisse avoir une fois pour toutes, le pas sur un autre ou le pas sur tous les autres.» [...] «En Europe [...], l'économie en développement rapide a pris assez. 
de marché puis le capitalisme se développent en invisibilisant une grande partie de la «civilisation matérielle » que Braudel nomme « économie du rez-de-chaussée ». Cet ordre économique n'est pas sans influence sur l'espace public. Tout d'abord, ce qui constitue le coeur actuel de l'espace public, les mass médias, obéit à une logique économique de profit et de concentration qui met souvent à mal la qualité des informations et le pluralisme de la presse. Ensuite, les acteurs économiques sont aussi, très souvent des acteurs politiques prenant positions dans l'espace public. C'est vrai de dirigeants d'entreprises devenus membres du gouvernement (T. Breton, F. Mer, C. Lagarde, etc.), de syndicalistes contestants la politique économique du gouvernement ou de responsables de secteurs économiques réclamant publiquement une autre politique fiscale. Enfin, même en dehors des périodes de crise aiguë, l'économie est un thème majeur de l'espace public. Les débats passionnés sur le référendum européen, les campagnes de communication de grands distributeurs appelant les citoyens à adopter des gestes plus écologiques, ou les discours des militants altermondialistes contre la globalisation financière sont autant de réalités empiriques indiquant l'importance des discours économiques dans nos démocraties ; ce qui ne veut pas dire, une fois de plus, que la société capitaliste est, par essence démocratique. Simplement, si la démocratie se caractérise par l'existence d'un espace public où est débattu l'intérêt général, la question de la production et de la répartition des richesses est, inévitablement, prise dans ce débat. Les discussions récurrentes sur le développement durable et la lutte contre l'exclusion en sont une illustration marquante. D'ailleurs, une des causes actuelles du rejet des élites politiques n'est-elle pas l'incapacité de ces dernières à réguler le système économique, à résoudre des problèmes économiques comme le chômage, ou la baisse du pouvoir d'achat (Scharpf, 1999) ? Pourtant, le coup de force opéré par les marginalistes consistant à créer, selon les mots de Walras, « une science économique pure » détachée de la science politique, a conduit à un partage disciplinaire qui empêche trop souvent les analystes non marxistes de saisir cette dimension économique de l'espace public.

souvent le pas sur les autres secteurs à partir $d u X^{e}$ ou $d u X I I^{e}$ siècle, plus sûrement encore à partir du XVI ${ }^{e}$; elle les a obligés à se définir par rapport à elle et cette primauté qui s'affirme, nul doute que ce ne soit une des racines de la modernité précoce de l'étroit continent » (T2, p. 409) 
La démocratie possède une double nature, c'est à la fois une société singulière qui se fonde sur elle-même et un régime politique particulier, marqué par l'existence d'un espace public en constante évolution. Du coup l'espace public doit être repensé dans toute sa complexité. Complexité politique comme nous y invite Habermas qui rejette le simplisme de la vision libérale, mais aussi complexité symbolique et économique, puisque l'espace public est l'instance de régulation, propre à la démocratie, des conflits entre l'ordre politique (élaboration de la norme), l'ordre symbolique (la circulation du croire) et l'ordre économique (la valorisation des ressources). Le but de cet article est donc de rappeler cette triple dimension de l'espace public. Car c'est en pensant ensemble les aspects politiques, économiques et symboliques de l'espace public que l'on peut penser la crise des démocraties européennes en dehors des poncifs tels que la crise de la représentation, l'aliénation économique des médias ou la perversion des sondages. 


\section{Bibliographie}

ARENDT, H., La Condition de l'homme moderne, Paris, Calmann-Levy, 1983.

BERLIN I. Eloge de la liberté, Paris, Calmann-Levy, 1969.

BLONDIAUX, L., LEVÊQUE S., « La politique locale à l'épreuve de la démocratie» in NEVEU, C., Espace public et engagement politique, Paris, L'Harmattan, 1999.

BRAUDEL F., Civilisation matérielle, Economie et capitalisme, trois tomes, Paris, Armand Colin, $2^{\grave{a}}$ ed, 1979.

CAIllé A. HUMBerT M., La démocratie au péril de l'économie, Rennes, PUR, 2006.

Calhoun, G. (dir.), Habermas and the Public Sphere, Cambridge Mass., MIT Press, 1992.

Castoriadis C., L'institution imaginaire de la société, Paris., Seuil, 1975

CHANIAL, P., Justice, don et association. La délicate essence de la démocratie, La Découverte, Mauss, Paris, 2001.

COTTEREAU, A., LADRIÈRE, P. (dir.), Pouvoir et légitimité. Figures de l'espace public, Paris, Éditions de l'École des hautes études en Sciences sociales, 1992.

DACHEUX, E (coord.), L'espace public, Paris, Cnrs éditions, 2008

DACHEUX, E (dir.), L'Europe qui se construit : réflexions sur l'espace public européen, Saint Étienne, Puse, 2003.

DAHLGREEN, P., "Media, citizens and civic culture" in M. Gurevitch and J. Curran eds, Mass Media and Society, London, Edward Arnold.

DAHLGREEN, P., Television and Public Sphere, Londres, Sage, 1995.

Eme B. «Agir solidaire et publicité des conflits », Hermès, N³6, 2003.

FLORIS, B., « Espace public et sphère économique », Hermès $N^{\circ} 36,2003$.

FLORIS, B., L'Entreprise dans l'espace public, Grenoble, PUG, 1996.

FORGUES E., « Vers un tournant symbolique post structuraliste en sciences sociales », Religioglogiques, N022, 2000.

FRANÇOIS, B., NEVEU, E., Espaces publics mosaïques, Rennes, PUR, 1999.

GARNHAM N., «Media and the public sphere », in Calhoun, G. (dir.), Habermas and the Public Sphere, Cambridge Mass., MIT Press, 1992.

HABERMAS, J., Droit et démocratie, Paris, Gallimard, 1997.

HABERMAS, J., L'Espace public, Paris, Payot, 1978.

HAYECK, F. VON, Droit, législation et liberté, Paris, PUF, 1986.

HERVIEU-LÉGER D. "La réligion mode de croire", MAUSS, N²2, 2003.

ION J., L'Engagement au pluriel, Saint Étienne, Puse, 2001.

ION J., La Fin des militants ?, Paris, Les Éditions de l'atelier, 1997.

LANE G., " Gary Becker et l'analyse économique des phénomènes sociaux », article paru en 1993 in Liberté économique et progrès social Nº7, 1993, consulté en juin 2006 sur http://blog.gerogeslane.fr

LAVILLE, J.-L., «Démocratie et économie : éléments pour une approche sociologique», Hermès $\mathrm{n}^{\circ} 36,2003$.

LECA, J., « La question démocratique » in DAMAMME, D. (dir.), La Démocratie en Europe, Paris, L'Harmattan, 2004.

LEFORT, C, Essai sur le politique, Paris, Seuil, 1986. 
LENOBLE, J., DEWANDRE, N. (dir.), L'Europe au soir du siècle. Identité et démocratie, Paris, Éditions Esprit, 1992.

MELTON, J. VAN HORN, The Rise of the Public in Enlightenment Europe, Cambridge, Cambridge University Press, 2001.

MERCIER, A. (dir.), Vers un espace public européen, Paris, l'Harmattan, 2003.

MulhmanN, G,. Le Regard du journalisme en démocratie, Université de Paris VII, thèse de science politique, 2001.

NEGT, O., L'Espace public oppositionnel, Paris, Payot, 2007.

NEVEU, C., Espace public et engagement politique, Paris, L'Harmattan, 1999.

PAILLART, I. (dir.), L'Espace public et l'emprise de la communication, Grenoble, Ellug, 1995.

POLANYI K., La grande transformation : Aux origines politiques et économiques de notre temps, Paris, Gallimard, 1983.

Prévost B., «Adam Smith : vers la fin d'un malentendu ? », L'économie politique, $\mathrm{N}^{\circ}$, 2001.

QUADERNI, n 18 , « Les espaces publics », Paris, L’Harmattan, 1992.

RANCIÈRE, J., La Haine de la démocratie, Paris, La Fabrique, 2005.

RÉSEAUX, n³4, «Autour d'Habermas », Paris, Cnet, 1989.

RICCEUR, P., L'Utopie et l'idéologie, Paris, Seuil, 1997.

ROULLEAU-BERGER, F., La Ville-intervalle. jeunes entre centre et banlieue, Paris, Méridiens-Klinncksieck, 1993.

ROUQUETTE, S., Vie et mort des débats télévisés, Bruxelles, De Boeck, 2002.

SCHARPF F., Governing in Europe, Efficient and Democratic, Oxford University Press, Oxford, 1999.

SCHUdSON, M., "Was There Ever a Public Sphere?" in CALHOUN, G., Habermas and the Public Sphere, Cambridge, Mass., MIT Press, 1992.

SCHUMPETER J.A., Capitalisme, socialisme et démocratie, Paris, Payot, 1969.

SENNETT, R. , Les Tyrannies de l'intimité, Paris, Seuil, 1979.

SIMMEL G., Le conflit, Paris, Circée, 2005.

TASSIN E., «Espace commun ou espace public ? », Hermès, №10, 1992.

TAROT C., «Les lyncheurs et le concombre ou de la définition de la religion quand même », MAUSS, $\mathrm{N}^{\circ} 22,2003$.

TAYLOR, C. «Quelle principe d'identité collective ? » in LENOBLE, J., DEWANDRE, N. (dir.), L'Europe au soir du siècle, Paris, Éditions Esprit, 1992.

TILLY, C., La France conteste 1600-1980, Paris, Fayard, 1986.

VERANT, J.-P., Les Origines de la pensée grecque, Paris, PUF, 1962.

WALZSER, M., « Sauver la société civile », Mouvement, $\mathrm{n}^{\circ}$ 8, 2000.

WEBER M., Economie et société, Paris, Plon, 1971.

WOLTON, D., La Dernière utopie, Paris, Flammarion, 1993.

WOLTON D., Il faut sauver la communication, Paris, Flammarion, 2005.

WUNENBURGER J-J. « Déclin et renaissance de l'imagination symbolique », Sociologie et sociétés, vol.XVII, 1985. 